
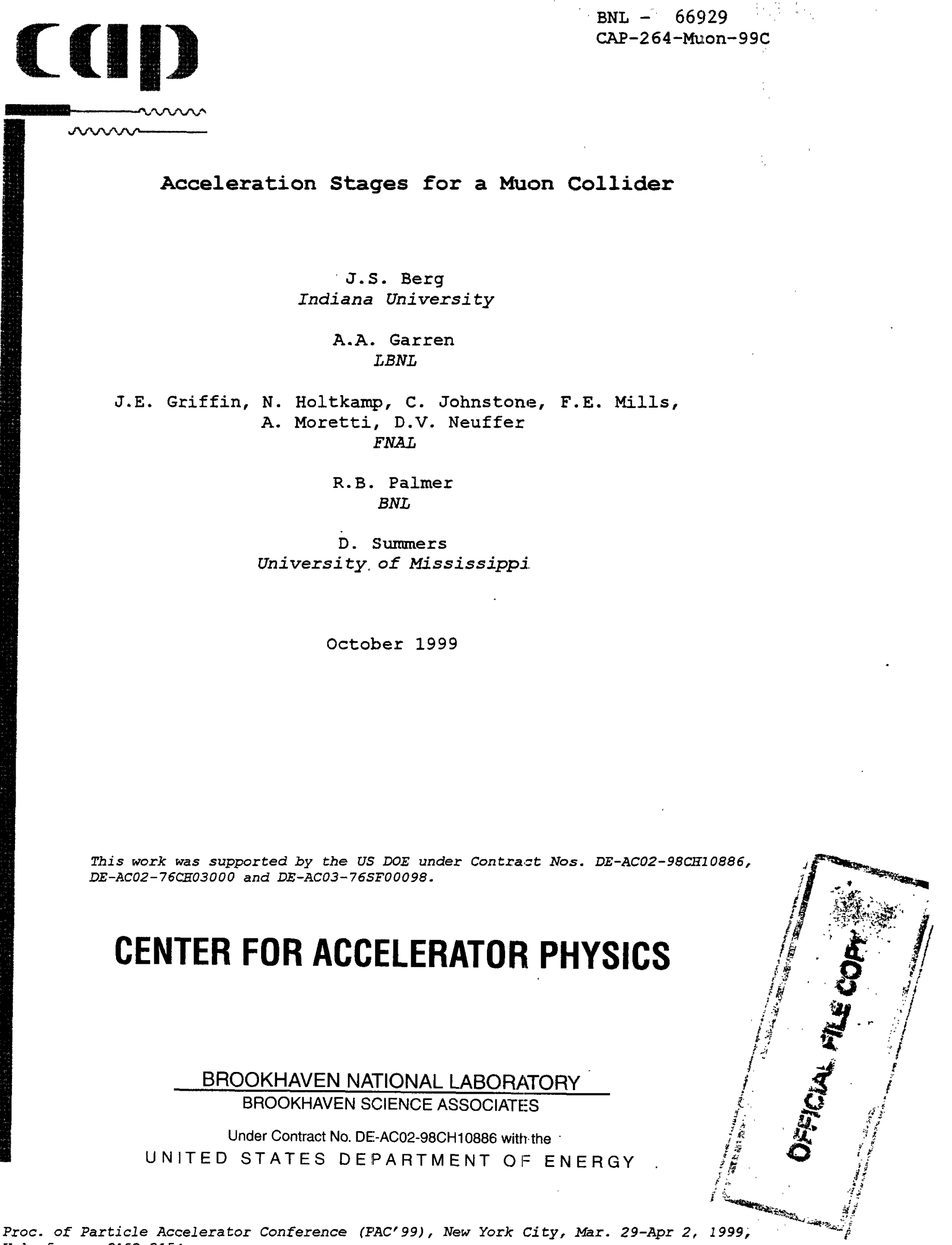



\title{
Acceleration Stages for a Muon Collider
}

\author{
J. S. Berg, Indiana U.; A. A. Garren, LBNL; \\ J. E. Griffin, N. Holtkamp, C. Johnstone, F. E. Mills, A. Moretti, D. V. Neuffer, FNAL; \\ R. B. Palmer, BNL; D. Summers, U. Mississippi
}

\begin{abstract}
Using muons in high energy colliders has an advantage in that the muons emit negligible synchrotron radiation, but has the disadvantage that the lifetime of the muons is very short. The latter requires that the muons be accelerated as rapidly as possible to prevent particle loss. The former allows one to use something other than a single long, straight linac to achieve that acceleration, and thereby achieve potentially significant cost advantages. Some of the acceleration systems will use recirculating linacs to achieve the reuse of the accelerating structures. However, the large longitudinal beam emittances make it difficult to simultaneously achieve large gradients by using high frequency RF and design an arc with a sufficient energy acceptance. The current state of the design for the acceleration stages for a muon collider will be presented, with emphasis on systems accelerating to $70 \mathrm{GeV}$ per beam. Known difficulties will be described as will their possible solutions. In particular, the use of an FFAG-like lattice for the arcs of a recirculating linac will be described which accepts a beam with a very large energy spread over a wide range of energies, allowing a single arc to be used instead of multiple arcs.
\end{abstract}

\section{INTRODUCTION}

This paper will describe the design issues and the current status of the acceleration stages for a first muon collider. In particular, it will focus on the design of systems to accelerate up to $70 \mathrm{GeV}$. While future machines will need to go to much higher energies, the lower energy acceleration systems will be necessary. One of the design criteria the acceleration systems for a $70 \mathrm{GeV}$ muon collider is that they could be reused with minimal modifications in a higher energy collider. Table 1 gives some of the parameters needed for the design of the acceleration stages.

\subsection{Overall Scheme}

We could conceivably get to $70 \mathrm{GeV}$ with a single linac. However, this fails to take advantage of the fact that the muons have negligible synchrotron radiation and can therefore follow a curved trajectory. Because of this, an acceleration system can be created where the muons pass through the linacs many times, potentially achieving significant cost savings. In doing this, one must take into account the additional decay losses when the muons are not being accelerated. This essentially eliminates the possibility of using ramped magnets in a synchrotron-like configuration at the
Table 1: Relevant parameters. Numbers are given for a low energy spread "Higgs factory" scenario and for a high energy machine. $N$ is the number of particles per bunch (after acceleration), $N_{\text {bunch }}$ is the number of bunches of each charge, $\epsilon_{6 n}$ is the 6-D normalized emittance (after acceleration), $\epsilon_{n}$ is the transverse normalized emittance (after acceleration), and $p_{\text {in }}$ is the momentum of the muons entering the acceleration systems.

\begin{tabular}{l|cc} 
& Higgs & High E \\
\hline \hline$N$ & $4 \times 10^{12}$ & $2 \times 10^{12}$ \\
$N_{\text {bunch }}$ & 2 & 4 \\
$\epsilon_{6 n},(\pi \mathrm{m})^{3}$ & \multicolumn{2}{|c}{$170 \times 10^{-12}$} \\
$\epsilon_{n}, \pi \mathrm{m}$ & $290 \times 10^{-6} 50 \times 10^{-6}$ \\
$p_{\text {in }}, \mathrm{MeV} / \mathrm{c}$ & \multicolumn{2}{|c}{186}
\end{tabular}

lower energies (the magnets would need to be ramped too quickly, or too much circumference of the ring would be wasted in arc).

The acceleration systems will be divided into several stages. As the beam energy increases, the (unnormalized) emittance decreases, allowing the use of higher frequency (and therefore higher gradient) RF and allowing the arcs to have a smaller energy acceptance. However, the required bending radius for the arcs increases with increasing energy. Thus, different types of acceleration systems will be used for different energy ranges, and different stages of a given type of acceleration system may be used in sequence (for instance, increasing the arc bending radius and increasing the RF frequency from one energy range to the next).

\subsection{Expected $R F$ parameters}

We're assuming the following relationships for the parameters of the r.f. cavities:

$r_{s}=40 \mathrm{M} \Omega / \mathrm{m} \sqrt{f / 800 \mathrm{MHz}}, Q=30000 \sqrt{800 \mathrm{MHz} / f}$, and $v_{\max }=30 \mathrm{MV} / \mathrm{m} \sqrt{f / 800 \mathrm{MHz}}$; here $r_{s}$ is the shunt impedance per unit length, $v_{\max }$ is the maximum peak gradient, and $f$ is the frequency. These are parameters which we expect to be reasonably achievable before the time that a muon collider is built.

\section{TTYPES OF STAGES}

Various types of systems have advantages and disadvantages which make them more or less suitable for various 
energy ranges.

\subsection{Pure Linac}

A linac has the advantage that it has no arcs, and therefore does not have the energy acceptance issues associated with arcs. There is still an energy acceptance associated with the height of the bucket, however. One disadvantage is that the particles make only one pass through such a system.

A linac is probably optimal for the lowest energies (up to $1 \mathrm{GeV}$, for example): it does not need to be particularly long, and the larger longitudinal emittance at the lowest energies is more readily accommodated by a linac.

In reality, several linacs would be required, since the requirements that the beam be matched longitudinally and fit inside the RF bucket require different frequencies at different energy ranges to achieve the optimal acceleration gradients (which is very important at these low energies due to the shorter muon lifetime). The following would be close to the optimal scheme to accelerate to $1 \mathrm{GeV}$ :

\begin{tabular}{r|rr} 
Energy range & Frequency & Length \\
\hline \hline $186-300 \mathrm{MeV} / \mathrm{c}$ & $50 \mathrm{MHz}$ & $20 \mathrm{~m}$ \\
$300-600 \mathrm{MeV} / \mathrm{c}$ & $100 \mathrm{MHz}$ & $40 \mathrm{~m}$ \\
$600-1000 \mathrm{MeV} / \mathrm{c}$ & $200 \mathrm{MHz}$ & $45 \mathrm{~m}$
\end{tabular}

The matching requirement requires that one runs rather far off crest (over $50^{\circ}$ ), reducing the gradient, and it should therefore be considered whether that is necessary or useful. Further study is required to determine whether the lowest frequencies above are realistic.

\subsection{Multiple Arc Recirculating Linac}

This design (used in CEBAF) has the advantage that several passes are made through the linacs, thereby getting a larger amount of acceleration from a given length of linac and amount of RF power. The primary difficulty with such a system is related to the larger longitudinal emittance of the beam. Since the RF frequency needs to be as high as possible to maximize the acceleration gradient, this leads to a relatively large energy spread in the beam. This energy spread, at least at lower energies, is often so large that $\pm 3 \sigma$ of the beam will not pass through a traditionally designed arc. In addition, the energy spread in the beam is too large to have passive switching into the appropriate arc, and so very fast kickers are required, which additionally must have larger strengths and transverse apertures.

One solution to avoid the cost of individual magnets for each arc is to have a single magnet with multiple bores that the beam can pass through, depending on the energy. The disadvantage of this is that one may lose turn-by-turn control of the path length and other parameters. Superconducting magnets with as many as 18 apertures with fields varying over the range of 0.7 to $7.0 \mathrm{~T}$ have been designed [1].

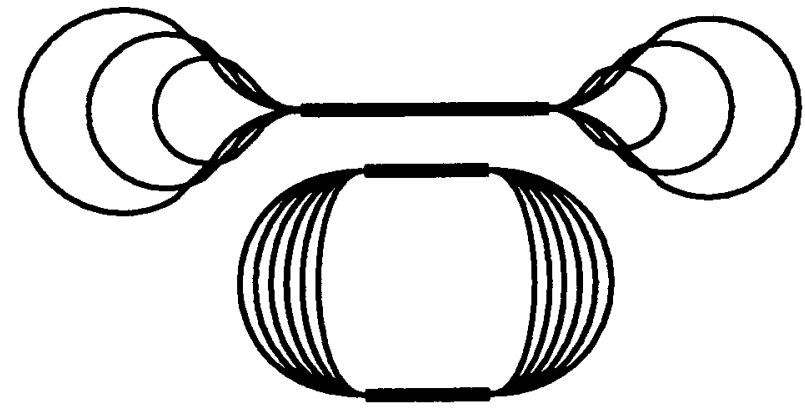

Figure 1: Comparable dogbone (above) and recirculating (below) geometries. Arcs are disproportionately long.

\subsection{FFAG Recirculator}

This is an attempt to address the issue of the energy acceptance of the arcs by making a single arc with an enormous energy acceptance; we have looked at designs for the 4$16 \mathrm{GeV}$ range and the $16-70 \mathrm{GeV}$ range. This additionally can avoid the need for kickers to switch between arcs, as well as allowing multiple beamlines and therefore multiple sets of magnets. There are several difficulties with such a system, however: when using only a single arc for all energies, one loses the ability to control parameters such as path length on a turn-by-turn basis, as well as the local momentum compaction; the arc design becomes much more complex; matching into straight sections becomes more difficult and can be done perfectly only for one energy; and the magnets are large and require high fields.

Two types of FFAG designs are being considered: a "scaling FFAG," where the orbits have the same geometric shape for all energies [2]; and a non-scaling variety which is generally more compact than the scaling FFAG.

One compromise solution may be to use a FFAG-like lattice to increase the energy acceptance of a multiple-arc recirculator.

\subsection{Dogbone}

As far as muon decays are concerned, any time in an arc is bad. In an racetrack recirculating linac design, the distance between the two linacs is determined by the radius of curvature required for the maximum energy. The minimum radius of curvature for lower energies is significantly smaller than the minimum radius at the maximum energy, and so if the linacs were closer, one could have a smaller distance traveled in the arcs. One solution to this problem is a "dogbone" scheme: the two linacs are placed in line with each other (instead of parallel), the beam goes through the two linacs, turns around, and enters the same linac that it just exited (see comparison in figure 1). The radius of curvature for the arcs can be as small as possible for the energy of each particular. Thus, there is a substantial reduction in the amount of time spent in arcs at the lower energies. Not only will this reduce the muon decays, but it can potentially 


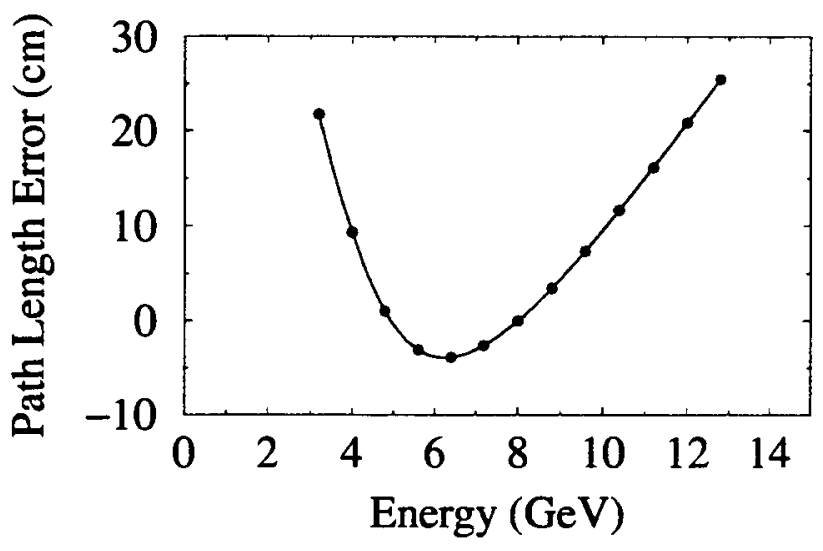

Figure 2: Path length as a function of energy for an FFAG arc. This is the error for a single turn, not a cumulative error; changing the RF frequency only changes where zero lies in this graph.

reduce the droop in $R F$ voltage due to wall losses, giving slightly higher effective gradients. At higher energies, the path length is actually slightly longer than it would be with an racetrack design, but it is the low energies which are critical for muon decays since the muon lifetime is shorter for lower energies. There could potentially be higher costs, however, since each arc would most likely require its own tunnel, and there would be no opportunity to use multiplebore magnets.

One promising use for the dogbone scheme is in an early stage of the acceleration scheme, where the energies are still relatively low. The dogbone could be designed so that the sequence that the beam sees is linac-arc-linac-arc-linac, eliminating the requirement for multiple arcs at each end (and the associated kickers), and yet using the same linac for three passes. This is currently our preferred solution for the $1-4 \mathrm{GeV}$ range; it would require a single $1 \mathrm{GeV}$ linac to accelerate from 1 to $4 \mathrm{GeV}$. Using $200 \mathrm{MHz} \mathrm{RF}$, we could hope to keep such a linac under $100 \mathrm{~m}$ long.

\section{SPECIFIC ISSUES}

\subsection{Dynamic RF Control}

With a recirculating system, there are difficulties in synchronizing the beam with the RF from one turn to the next. This is especially important for the FFAG arcs, since the path length cannot be controlled on individual turns, and the path length varies substantially with energy (see figure 2). In addition, there will be a variation in the beam loading (and therefore the output energy of the acceleration stages) with the bunch current, which will not be known a priori to high accuracy. To correct for this, some kind of dynamic control of the RF systems is necessary, which may require that at least the phase of the RF can be changed (maybe substantially) from one turn to the next.

There are three potential ways to do this: one can pump in power, one can use ferrites which have their permit- tivity changed dynamically (changing the RF frequency), or one can create a path length variation using fast magnets. Pumping in power to correct phase errors like those in figure 2 could potentially double the peak power requirements, and thus is an undesirable solution (but may be possible for correcting variations from beam current fluctuations). Ramping magnets sufficiently quickly to achieve substantial path length variations in the smaller rings (such as a $300 \mathrm{~m}$ long $4-16 \mathrm{GeV}$ FFAG) is probably impossible. Thus, using ferrites or some other mechanism to dynamically change the RF frequency is an attractive solution. Whether it is possible to do this with these types of RF systems is being studied.

\subsection{Kickers}

For injection and extraction, as well as for switching between arcs in any of the multiple-arc recirculation schemes, we require kickers which are both fast and have a large transverse acceptance.

The case giving the most stringent requirements for a kicker will probably be a recirculating linac or FFAG in the 4-16 GeV range: the circumference is the smallest of all the recirculating-type machines (around $300-400 \mathrm{~m}$ ), and the beam emittance will be at its largest (amongst the recirculating machines). Taking a $\beta$-function of $50 \mathrm{~m}$, and a ring circumference of $300 \mathrm{~m}$, the requirements for such a kicker at $4 \mathrm{GeV}$ would be approximately

$\begin{array}{lr}\text { Aperture (full width) } & 12 \mathrm{~cm} \\ \text { Kick strength } & 43 \mathrm{mT} / \mathrm{m} \\ \text { Rise tirne (full) } & 500 \mathrm{~ns} \\ \text { Repetition time } & 60 \mathrm{~ms}\end{array}$

From table 1 in [3], these appear to be reasonably achievable requirements (that table gives significantly longer repetition times, but there is no reason to believe that those designs could not be run at higher repetition rates). Up to a point, the $\beta$-function can be reduced to reduce the required aperture at the cost of increasing the required kick strength. The required rise time increases to $1000 \mathrm{~ns}$ if the kickers are only required for injection and extraction.

\subsection{Other Effects}

There are other effects which have not been discussed here; amongst them are HOM wakefield effects, the effect of having two beams, and magnet technology necessary for the arcs (particularly the FFAG's).

\section{REFERENCES}

[1] S. Khan, G. Morgan, and E. Willen, "Recirculating arc dipole for the $2+2 \mathrm{TeV} \mu^{+}-\mu^{-}$Collider," unpublished (1996).

[2] K. R. Symon et ai., "Fixed-Field Alternating-Gradient Particle Accelerators," Phys. Rev. 103, 1837-1859 (1956).

[3] G. H. Schröder, "Fast Pulsed Magnet Systems," CERN-SL98-017, to appear in Handbook of Accelerator Physics and Engineering, edited by A. Chao and M. Tigner, World Scientific (1999). 
